American Journal of Applied Sciences 5 (10): 1344-1347, 2008

ISSN 1546-9239

(C) 2008 Science Publications

\title{
Electromagnetic Assessment on Human Safety of Mobile Communication Base Stations at University of Macau
}

\author{
K.H. Chiang and K.W. Tam \\ Wireless Communication Laboratory, Faculty of Science and Technology, \\ University of Macau, Av. Padre Tomas Pereira, Taipa, Macao SAR, China
}

\begin{abstract}
Assessment of the electromagnetic exposure due to the mobile communication base station at University of Macau is presented by two testing scenarios. The first scenario about 20 in-situ measurements and the maximum E-field strength was $2.46 \mathrm{~V} / \mathrm{m}$ that well below the threshold and compliant to the safety guideline. The second scenario investigates on the distance effect of exposure due to the base station and it is found that the field strength decays by $0.12 \mathrm{~dB} / \mathrm{m}$. Thus, low radiation level estimated at the university when compared with the measured values.
\end{abstract}

Key words: Radiation safety, Specific Absorption Rate (SAR), International Commission on NonIonizing Radiation Protection (ICNIRP)

\section{INTRODUCTION}

In the past few decades, the mobile communication has been evolved from analogue system to latest digital cellular communications. In Macau where is a small city with an area of around $28 \mathrm{Km}^{2}$ and located $60 \mathrm{Km}$ from Hong Kong, its mobile communication also evolved from GSM 900 in 1995 to GSM 1800 and CDMA in 1999 and 2006 respectively. There are totally four public digital cellular communication operators in Macau and over 635,000 subscribers registered in 2006. This rapid development has also brought the persistent public concerns about the possible adverse health effects of electromagnetic emissions from digital cellular telephone base stations. It is thus necessary to carry out the electromagnetic compatibility assessment to identify whether the level of emission due to the base stations represents a risk for human health according to the international safety guideline. In particular, there is a digital cellular base station located in the hostel area nearby the central campus of University of Macau as shown in Fig. 1a. As illustrated, the base station antenna is installed in the rooftop of hostel building Block II as in Fig. 1b and these antennas are facing the central campus building named Choi Kai Yau shown in Fig. 1c where more than 35 classrooms and 40 office rooms are allocated. The base station is generally emitting the RF power of $42 \mathrm{dBm}$ and $38 \mathrm{dBm}$ for GSM 900/1800 and CDMA respectively. The purpose of this work was mainly to perform in-situ

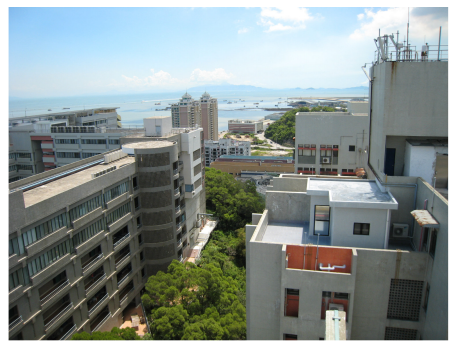

a

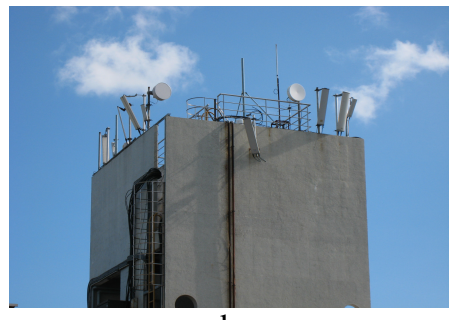

b

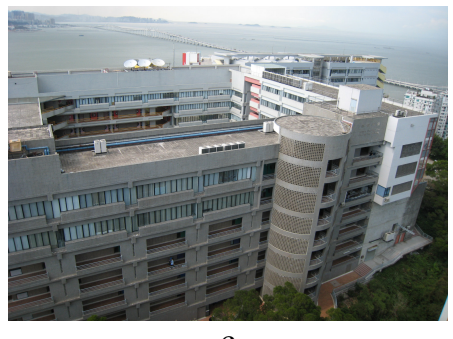

C

Fig. 1: a Base station nearby Choi Kai Yau building, (b) A base station in the roof of block II building, (c) Choi Kai Yau building in the central campus

Corresponding Author: K.W. Tam, Wireless Communication Laboratory, Faculty of Science and Technology, University of Macau, Av. Padre Tomas Pereira, Taipa, Macao SAR, China Tel: (853) 3974496 Fax: (853) 28838314 
electromagnetic field strength measurement of base station at University of Macau in order to evaluate the radiation safety compliance for GSM 900/1800 and CDMA mobile communication base stations. Two testing scenarios were used to characterize the radiation exposure due to the base station according to the ICNIRP (International Commission on Non-Ionizing Radiation Protection) safety guideline ${ }^{[1]}$.

\section{MATERIALS AND METHODS}

To evaluate the electromagnetic exposure, the used radiation safety standard is summarized together with the discussion of the wide band field strength meter as follows.

Safety standard: There are some international radiation exposure standards available in Europe and America, that are mainly defined from the thermal point of view. These standards provide information on radiation exposure limits and assessment methods ${ }^{[2,3]}$. Amongst them, ICNIRP is widely adopted in many European countries and some Asian metropolis. To characterize the radiation absorbed by the human, the parameter used is the Specific Absorption Rate (SAR), which represents the rate at which electromagnetic energy is absorbed by unit of tissue mass. For examples, the general public exposure limit of whole body average SAR is $0.08 \mathrm{~W} \mathrm{~kg}^{-1}$ whilst the localized $\mathrm{SAR}$ for head and trunk is $2 \mathrm{Wkg}^{-1[4,5]}$. In fact, the above SAR value is difficult to measure as it must be obtained inside the body ${ }^{[6-9]}$. As an engineering exposure threshold, the reference level is established in terms of the electromagnetic quantities like power density, electric and magnetic field intensities, which are easily measured outside the body. For far field measurement, SAR is thus expressed in terms of the above parameters as follows ${ }^{[8]}$ :

$$
\mathrm{SAR}=\frac{\sigma|\mathrm{E}|^{2}}{\rho_{\mathrm{md}}}
$$

where, the electric field $\mathrm{E}$ is in $\mathrm{V} / \mathrm{m}, \sigma$ as the conductivity $\left(\mathrm{S} \mathrm{m}^{-1}\right)$ and the mass density $\rho_{\mathrm{md}}\left(\mathrm{kg} \mathrm{m}^{-3}\right)$. To assess the radiation safety ${ }^{[10-12]}$ in the frequency range of base stations in Macao, the reference levels in field strength are plotted against the frequency as in Fig. 2. The reference levels are ranged from 38.8 to $59.8 \mathrm{~V} \mathrm{~m}^{-1}$ deriving from the basic restricted SAR values.

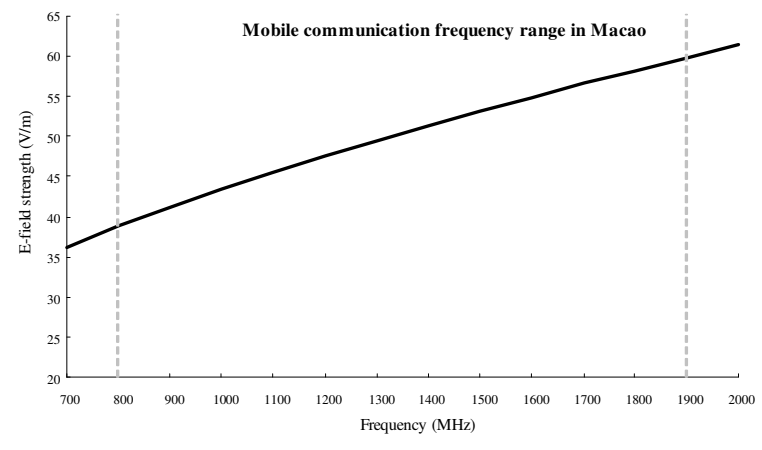

Fig. 2: Reference levels against frequencies

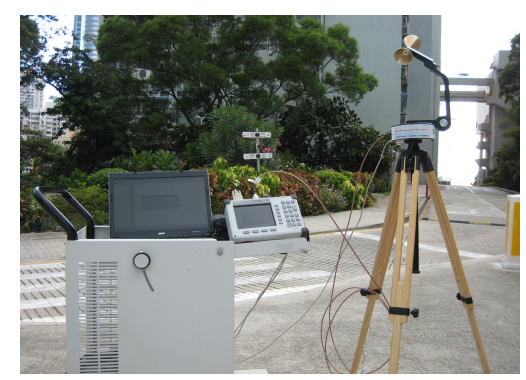

Fig. 3: In-situ measurement setup

In-situ measurement setup: For the safety compliance of electromagnetic exposure due to the base station in the campus, a portable spectrum analyzer connected with a wide band probe is used as shown in Fig. 3 .

This probe mounted on an automatic antenna rotator is constructed by precision conical dipole antenna with a holder aligning in three orthogonal directions and, the frequency is operated from $80 \mathrm{MHz}$ to $2.5 \mathrm{GHz}$. A laptop is also used to retrieve the field strength information from the spectrum analyzer and control measurement duration of 6 minutes and testing frequency range. Using this setup, the sensitivity in measured field strength is $0.001 \mathrm{~V} \mathrm{~m}^{-1}$. If the measured field strength exceeds the reference level, detailed spectrum analysis in the individual channel is thus investigated.

\section{RESULTS AND DISCUSSION}

Based on the above methodology, two exposure evaluation scenarios were performed. The first scenario is about localized measurement, i.e. twenty different locations were chosen inside the campus considering the dense human flow and the time spent by the students or staffs in a particular area. These locations 
Am. J. Applied Sci., 5 (10): 1344-1347, 2008

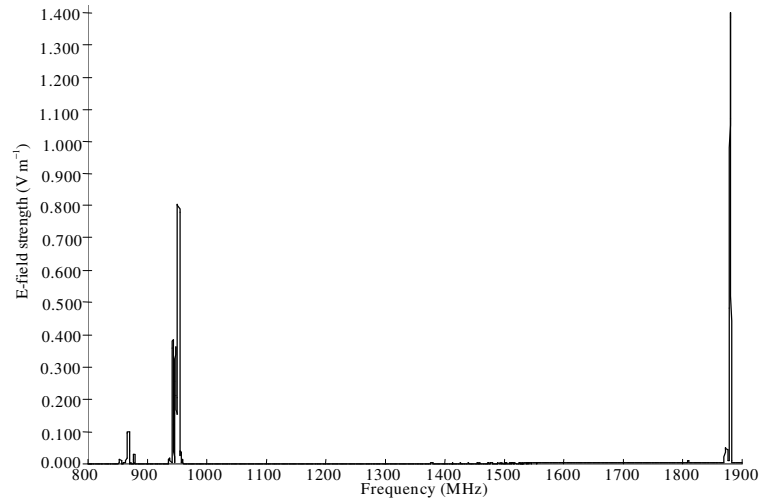

Fig. 4: The typical field strength for GSM 900/1800 and CDMA base stations

are divided into the hostel, classroom, office and outdoor. The second scenario was designed based on the testing location away the base station in the similar altitude, i.e. 40, 80, 120 and $160 \mathrm{~m}$. For the concerned base station, its basic spectrum spanned from $800 \mathrm{MHz}$ to $1.9 \mathrm{GHz}$ was measured and plotted in Fig. 4 .

It is observed that there were three principal electric field strengths located at GSM 900/1800 and CDMA bands. The field strengths of the above bands were thus calculated as $0.8,1.38$ and $0.1 \mathrm{~V} \mathrm{~m}^{-1}$ respectively. These values were well below the corresponding reference levels and compliant to the safety guideline.

First scenario: Twenty campus locations attributed by their latitudes and longitudes, temperatures in measurement were selected for electromagnetic exposure evaluation. These locations are categorized as hostel, classroom, office and outdoor. The strengths at these locations were measured and, their minimum and maximum values were 0.048 and $2.46 \mathrm{~V} \mathrm{~m}^{-1}$ respectively, accounting for a difference of $34.2 \mathrm{~dB}$. The maximum electric field strength occurred in the roof of Choi Kai Yau Building (as in Fig. 1c) which is around $40 \mathrm{~m}$ in front of the base stations' antennas. Based on these field strengths, the minimum and maximum SAR values for human brain are estimated as $2.58 \mu \mathrm{W} \mathrm{kg}{ }^{-1}$ and $6.77 \mathrm{~mW} \mathrm{~kg}^{-1}$ given $1.1531 \mathrm{~S} \mathrm{~m}^{-1}$ for the conductivity and $1030 \mathrm{Kg} \mathrm{m}^{-3}$ as the mass density of the tested tissue ${ }^{[13]}$. The above measurement statistics was also studied in Fig. 5 in which, $15 \%$ of measurements were within the field strength range of 0.8 and $2.8 \mathrm{~V} \mathrm{~m}^{-1}$ whilst $30 \%$ were for the range of 0.3 to $0.8 \mathrm{~V} \mathrm{~m}^{-1}$, the majority $55 \%$ were below $0.3 \mathrm{~V} \mathrm{~m}^{-1}$.

The E-field strength variation at hostel, office, classroom and outdoor was also presented in Fig. 6. As

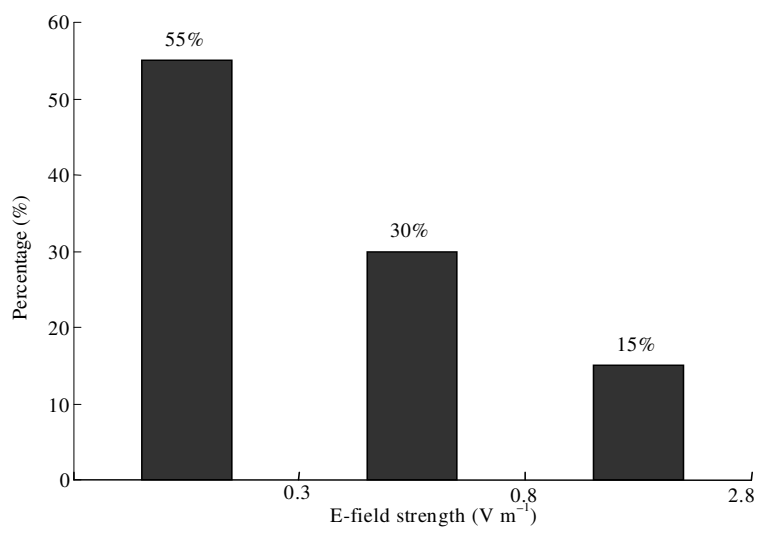

Fig. 5: The field strength distribution of first scenario

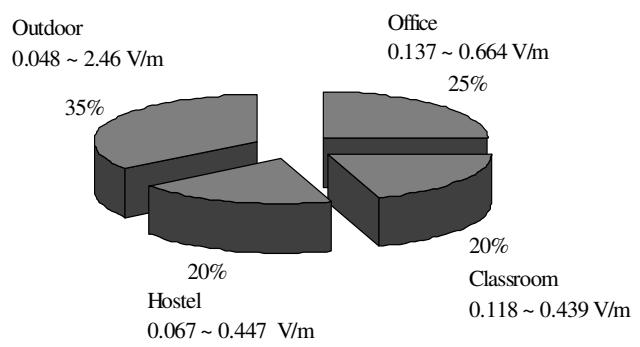

Fig. 6: The statistics of field strength in category

observed, the areas like classrooms and offices where students and staffs frequently stay, reported the strength level well below the corresponding reference levels and compliant to the safety guideline.

Second Scenario: To assess the radiation due to the base station in the central campus along the distance, an additional scenario was performed as follows. 6 outdoor locations were chosen and they were apart from the base station around 40, 80, 120 and $160 \mathrm{~m}$ along the same radial line. Field strengths were then measured at the above locations so as to explore the distance effect on electromagnetic exposure.

The minimum and maximum values were $0.1175 \mathrm{~V}$ $\mathrm{m}^{-1}$ at $151 \mathrm{~m}$ and $2.46 \mathrm{~V} \mathrm{~m}^{-1}$ at $40 \mathrm{~m}$ respectively, accounting for a difference of $26.4 \mathrm{~dB}$. The corresponding minimum and maximum SAR values for human brain are estimated using the same tissue parameters in the last sub section and they were $37 \mu \mathrm{W}$ $\mathrm{kg}^{-1}$ and $6.77 \mathrm{~mW} \mathrm{~kg}^{-1}$ respectively. As shown in Fig. 7, this distance effect was plotted and it is obvious that the field strengths varied $0.12 \mathrm{~dB}$ per meter along the location distanced from the base station. The above strengths were again well below the corresponding reference levels and compliant to the safety guideline. 


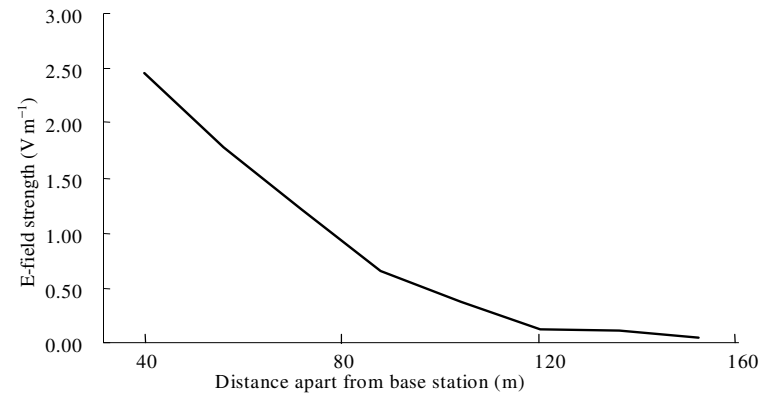

Fig. 7: Field strengths variation against the distance apart from the base station

\section{CONCLUSIONS}

In this work, the radiation exposure due to the base station at University of Macau was evaluated by two scenarios. The first scenario was about 20 localized measurements. Amongst them, no measurements were found against the safety reference value and the majority around $55 \%$ measurements were below $0.3 \mathrm{~V}$ $\mathrm{m}^{-1}$. The second scenario was designed based on the testing location apart from the base station. Like the first scenario, no measurement exceeded the reference level. The field strength decayed $0.12 \mathrm{~dB} \mathrm{~m}^{-1}$ as observed. Based on these two scenarios, the radiation due to the base station is compliant to the safety guideline.

\section{ACKNOWLEDGMENT}

This work was financially supported by the Science and Technology Development Fund of Macao project no. 042/2006/A3 and University of Macau Research Committee project no. RG060/06-07S/TKW/FST. In addition, authors would like to thank Mr. C.P. Chiang for the field strength measurements assistance.

\section{REFERENCES}

1. ICNIRP (International Commission on NonIonizing Radiation Protection), 1998. Guidelines for limiting exposure to time-varying electric, magnetic, and electromagnetic fields (up to 300 GHz), Health Physics, 74: 494-522.

2. International Commission on Radiation Units and Measurements, 1980. Radiation Quantities and Units, ICRU Report 33 (Bethesda, MD: ICRU).
3. Electronics Communications Committee (ECC) within the European Conference Postal and Telecommunications Administrations. Measuring Non-Ionizing Electromagnetic Radiation (9 kHz$300 \mathrm{GHz}$ ), October 2003.

4. Johnson, C.C., and A.W. Guy, 1972. Non-ionizing electromagnetic wave effects in biological material and systems, IEEE Proceedings, 60: 692-718.

5. Specific Absorption Rate (SAR) Estimation for Cellular Phone (Standard Version 2), ARIB STDT56, 2002.

6. Human Exposure to Radio Frequency Fields From Handheld and Body-Mounted Wireless Communication Devices-Human Models, Instrumentation and Procedures, Part 1: Procedure to Determine the Specific Absorption Rate (SAR) for Handheld Devices Used in Close Proximity to the Ear (Frequency Range of $300 \mathrm{MHz}$ to $3 \mathrm{GHz}$ ), IEC 62209 Part 1, 2005.

7. IEEE Recommended Practice for Determining the Peak Spatial-Average Specific Absorption Rate (SAR) in the Human Head From Wireless Communications Devices: Measurement Techniques, IEEE Standard 1528-2003, 2003.

8. Basic Standard for the Measurement of Specific Absorption Rate Related to Human Exposure to Electromagnetic Fields From Mobile Phones (300 MHz-3 GHz), CENELEC EN 50361: 2001, 2001.

9. Lin J.C., 2002. Radio frequency radiation safety and health, Radio Sci. Bull., 303: 37-39. [IEEE1887].

10. Drossos, A., V. Santomaa and N. Kuster, 2000. The dependence of electromagnetic energy absorption upon human head tissue composition in the frequency range of $300-3000 \mathrm{MHz}$, IEEE Trans. Microwave. Theory Tech., 48 (11): 1988-1995.

11. Ghandi, 1990. Biological Effects and Medical Applications of Electromagnetic Energy. Englewood Cliffs: NJ, Prentice Hall.

12. Guy, A.W. and C.K Chow, 1986. Specific Absorption Rates of Energy in Man Models Exposed to Cellular UHF Mobile-Antenna Fields. IEEE Trans on Microwave Theory Tech, vol. MTT-34.

13. Federal Communications Commission (FCC), 2006. FCC web site on Tissue Dielectrics. http://www.fcc.gov/fcc-bin/dielec.sh. 